\title{
Adipose-Derived Mesenchymal Stem Cells for the Treatment of Articular Cartilage: A Systematic Review on Preclinical and Clinical Evidence
}

\author{
Francesco Perdisa, ${ }^{1}$ Natalia Gostyńska, ${ }^{1}$ Alice Roffi, ${ }^{2}$ Giuseppe Filardo, ${ }^{1}$ \\ Maurilio Marcacci, ${ }^{1}$ and Elizaveta Kon ${ }^{2}$ \\ ${ }^{1}$ II Clinic, Biomechanics Laboratory, Rizzoli Orthopaedic Institute, 40136 Bologna, Italy \\ ${ }^{2}$ II Clinic, Nano-Biotechnology Laboratory, Rizzoli Orthopaedic Institute, 40136 Bologna, Italy
}

Correspondence should be addressed to Francesco Perdisa; francesco.perdisa@ior.it

Received 26 October 2014; Revised 29 December 2014; Accepted 28 January 2015

Academic Editor: Abhijit De

\begin{abstract}
Copyright (C) 2015 Francesco Perdisa et al. This is an open access article distributed under the Creative Commons Attribution License, which permits unrestricted use, distribution, and reproduction in any medium, provided the original work is properly cited.
\end{abstract}

\begin{abstract}
Among the current therapeutic approaches for the regeneration of damaged articular cartilage, none has yet proven to offer results comparable to those of native hyaline cartilage. Recently, it has been claimed that the use of mesenchymal stem cells (MSCs) provides greater regenerative potential than differentiated cells, such as chondrocytes. Among the different kinds of MSCs available, adiposederived mesenchymal stem cells (ADSCs) are emerging due to their abundancy and easiness to harvest. However, their mechanism of action and potential for cartilage regeneration are still under investigation, and many other aspects still need to be clarified. The aim of this systematic review is to give an overview of in vivo studies dealing with ADSCs, by summarizing the main evidence for the treatment of cartilage disease of the knee.
\end{abstract}

\section{Introduction}

Alterations in articular cartilage can produce pain and reduce the quality of life, evolving into the development of osteoarthritis (OA) and consequently permanent disabling symptoms.

Pharmacological or surgical treatments currently used for OA patients can help with the management of symptoms and delay disease progression [1-3], but metal resurfacing is the only currently available treatment able to provide pain relief and satisfactory function recovery, when the final stages of OA are reached [4-7]. Although new treatment options have come from recent achievements in biotechnologies $[8,9]$, the regeneration of hyaline cartilage is still a chimera [10]. Looking for a new direction in cartilage regeneration, the recent history of orthopaedics has been enriched with innovative therapies, ranging from platelet-derived growth factors (GFs) to cell-based treatments [11, 12], also combined with various biomaterials for tissue engineering strategies. In particular, recently mesenchymal stem cells (MSCs) are emerging as an alternative to the use of differentiated chondrocytes, thanks to their potential to differentiate into several lines such as osteoblasts, chondrocytes, myoblasts, or adipocytes and to their capability of self-renewal, high plasticity, and immunosuppressive and anti-inflammatory action [13]. MSCs can be obtained from different human sources, such as bone marrow, periosteum, umbilical cord blood, dermis, muscle, infrapatellar fat pad, synovial membrane, and adipose tissue [13]. Among these sources, adipose-derived mesenchymal stem cells (ADSCs) are attracting attention as an alternative to the better studied bone marrow mesenchymal stem cells (BMSCs) $[14,15]$. The reasons for increased interest in ADSCs reside in their abundance (ADSCs are $5 \%$ of nucleated cells versus $0.0001-0.01 \%$ of BMSCs), the ease with which they can be harvested (with the advantages of lower donor-site morbidity), and their rapid expansion and high proliferation potential [16]; moreover, they have shown that they can maintain their phenotype better over many culture passages with respect to BMSCs [17]. Thus, over the last decade, the number of preclinical and clinical papers dealing with ADSCs has 
increased significantly. However, their mechanism of action is not fully understood, since both the differentiation of stem cells by themselves and paracrine and trophic effects might be involved. Moreover, the optimal strategy for applying ADSCs has not yet been identified and many aspects still remain controversial [18].

The aim of this systematic review is to analyze the current literature on the use of ADSCs in vivo to show the available evidence on their therapeutic potential for cartilage regeneration, by investigating their efficacy, drawbacks, and possible future application strategies in humans for the treatment of chondral pathologies.

\section{Materials and Methods}

A systematic review of the literature was performed on the use of ADSCs for knee cartilage defects in vivo. The search was made using the PubMed database, by focusing on both preclinical and clinical studies, with the following thread: "cartilage" AND "adipose-derived mesenchymal stem cells" OR "adipose-derived stem cells" OR "adipose derived stromal cells" OR "stromal vascular fraction." The filters included publications from the last 10 years in the English language. Articles were first screened by title and abstract. Subsequently, the full texts of the resulting articles were screened and those not reporting the in vivo use of ADSCs or applications different from knee cartilage defects or OA were excluded. Cell sources other than adipose tissue were rejected unless they were used for comparative studies. All the articles dealing with applications of ADSCs different from those described above were also excluded. Reference lists of the selected articles were also screened to obtain further studies for this review.

\section{Results}

Following the above-mentioned screening procedure, 361 articles were initially found. Thirty-nine studies, meeting the above-mentioned criteria, were included in the final analysis: 28 animal studies (25 preclinical and 3 clinical) and 11 clinical studies. Among preclinical studies, 16 described arthrotomic implantation as a surgical procedure to treat focal osteochondral defects and 8 studies described intraarticular injections for an OA model. A further study evaluated 2 treatment groups for induced OA: by either injective or surgical administration. Culture-expanded ADSCs were used in all studies except one, where cells were isolated from adipose tissue by enzymatic digestion. A single study compared both methods. In 4 cases, expanded cells were manipulated by introducing different genes.

Among the clinical studies, 11 papers dealing with the administration of cells obtained from adipose tissue for the treatment of knee articular cartilage were included. Only one study described expanded ADSCs in the clinical setting, whereas all other cases reported on cells isolated by the enzymatic digestion of fat tissue.

The articles are described in detail in Tables 1 and 2, and the different aspects evaluated are summarized and discussed in the following paragraph.

\section{Discussion}

Over the last decade, an increasing number of papers have been published on the use of ADSCs for the treatment of cartilage disease, ranging from preclinical studies to clinical trials in the most recent years. In fact, adipose tissue is an appealing source of MSCs in the clinical setting, being an easy and abundant source for their harvest, which can be performed through a minimally invasive procedure, thus allowing a large number of cells to be obtained with minimal manipulations.

The studies analyzed in this systematic review present a variety of applications: cells can be used as a suspension after being cultured or freshly isolated by the enzyme-digestion of adipose tissue, thus obtaining a heterogeneous "stromal vascular fraction" (SVF) that contains a variety of stem, progenitor, and adult cells. Among them, it has been shown that ADSCs represent up to $10 \%$ of SVF [12]. The delivery can be performed by intra-articular injections, where cells are often suspended in a volume of platelet-rich plasma (PRP), or by surgical implantation, possibly by seeding them onto biomaterials, in the form of three-dimensional scaffolds.

Among the several papers analyzed, one of the first aspects to be underlined is the discrepancy between the approach used for their administration in the preclinical model and the modality actually predominant in the clinical setting. Preclinical trials used different animal models (16 rabbits, 3 mice, 1 rat, 2 pigs, 1 goat, 1 horse, and 1 sheep) and either expanded ADSCs (23) or SVF (1) were applied, plus 1 study that compared both methods. Cells were chondrogenicinduced (5) or noninduced (20), treated with GFs derived from platelets (2), or manipulated by introducing inductive genes (2). Conversely, the literature shows a different trend in the clinical application, both in terms of cell processing and delivery techniques: in fact, enzyme-isolated cells, in the form of SVF, were used in 13 of the 14 clinical studies (3 dog and 11 human), probably due to ease of manipulation and lower costs of a one-step procedure. This relies on the relatively high number of cells obtained from fat tissue with respect to other sources. Moreover, in all except one clinical study, cells were administered by intra-articular injection, whereas only one study reports the results of SVF surgical implantation, using fibrin glue as a scaffold for the treatment of isolated lesions. In fact, injective administration presents several advantages: it is minimally invasive, it has better patient compliance, and costs are lower [19]. Moreover, the rationale of this approach is to target not only the articular cartilage but also the whole joint environment, which is likely to be involved especially in degenerative diseases like OA [20,21]. Most of the studies described cell delivery following arthroscopic debridement and aimed at maximizing the benefits of both approaches.

\section{Preclinical Application}

Since the preclinical setting allows researchers to focus better on specific issues, some interesting findings emerged with regard to the different aspects of using ADSCs. In the following paragraphs, we have summarized the main evidence and indications that emerged from the systematic literature analysis. 


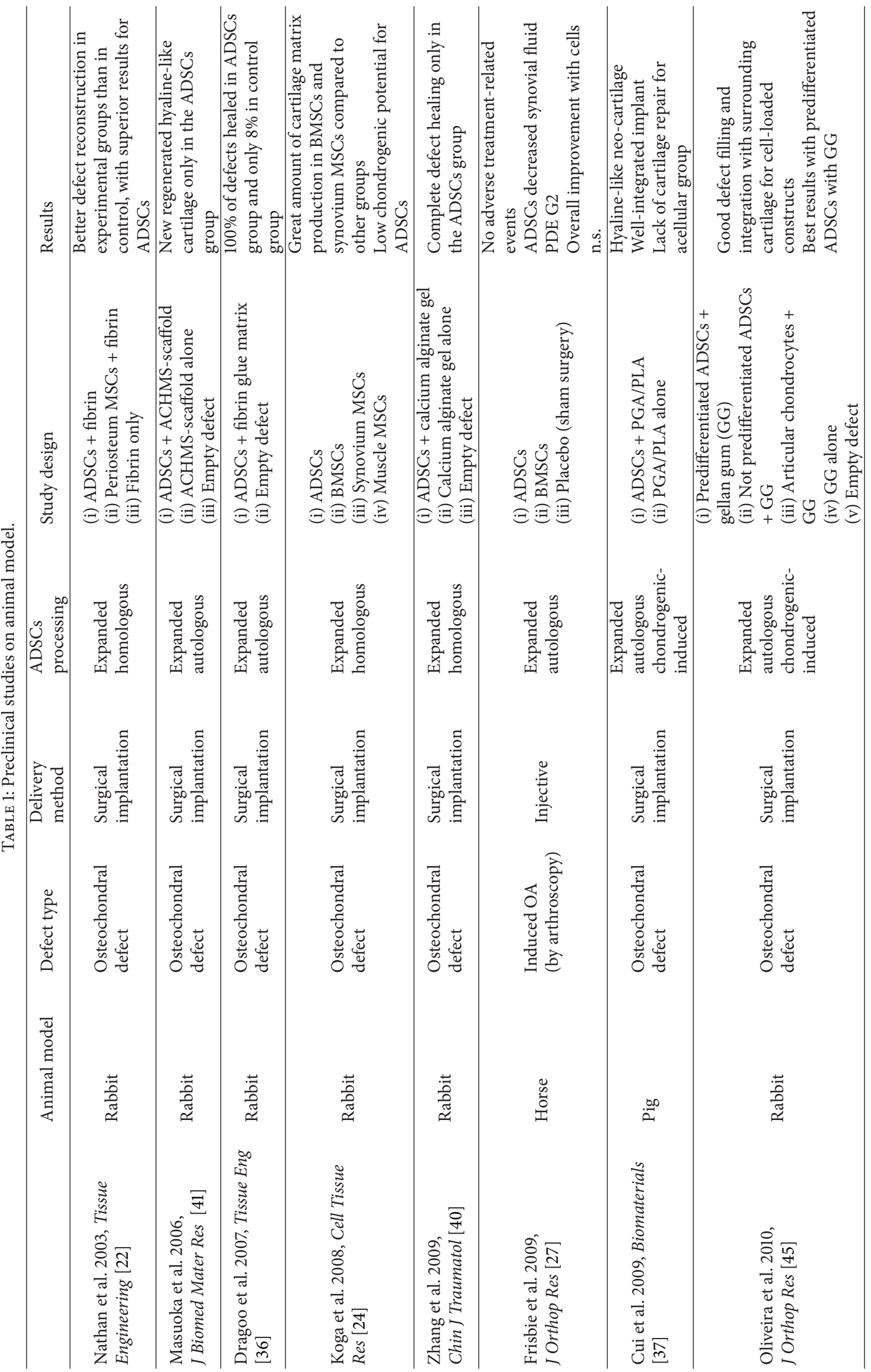




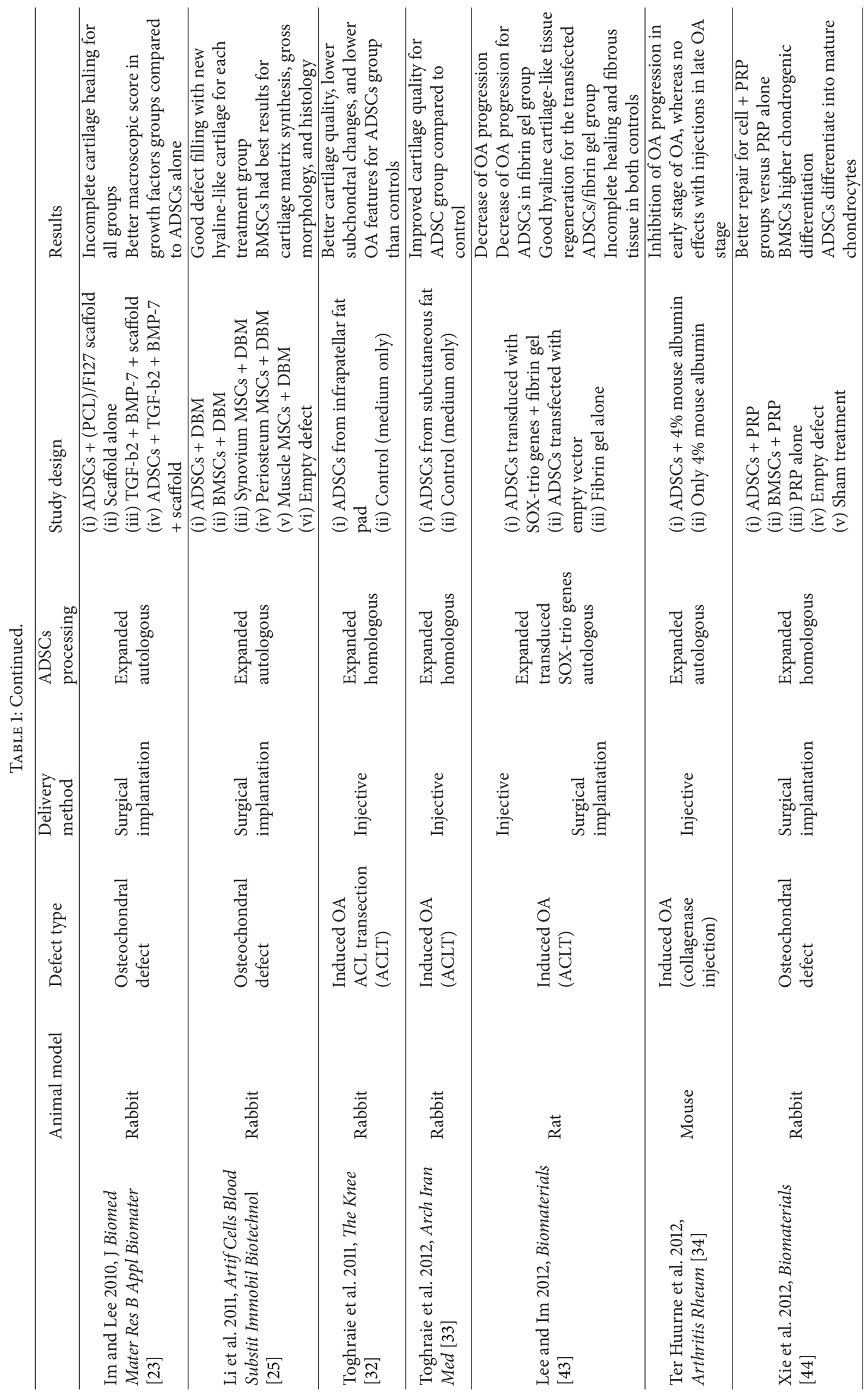




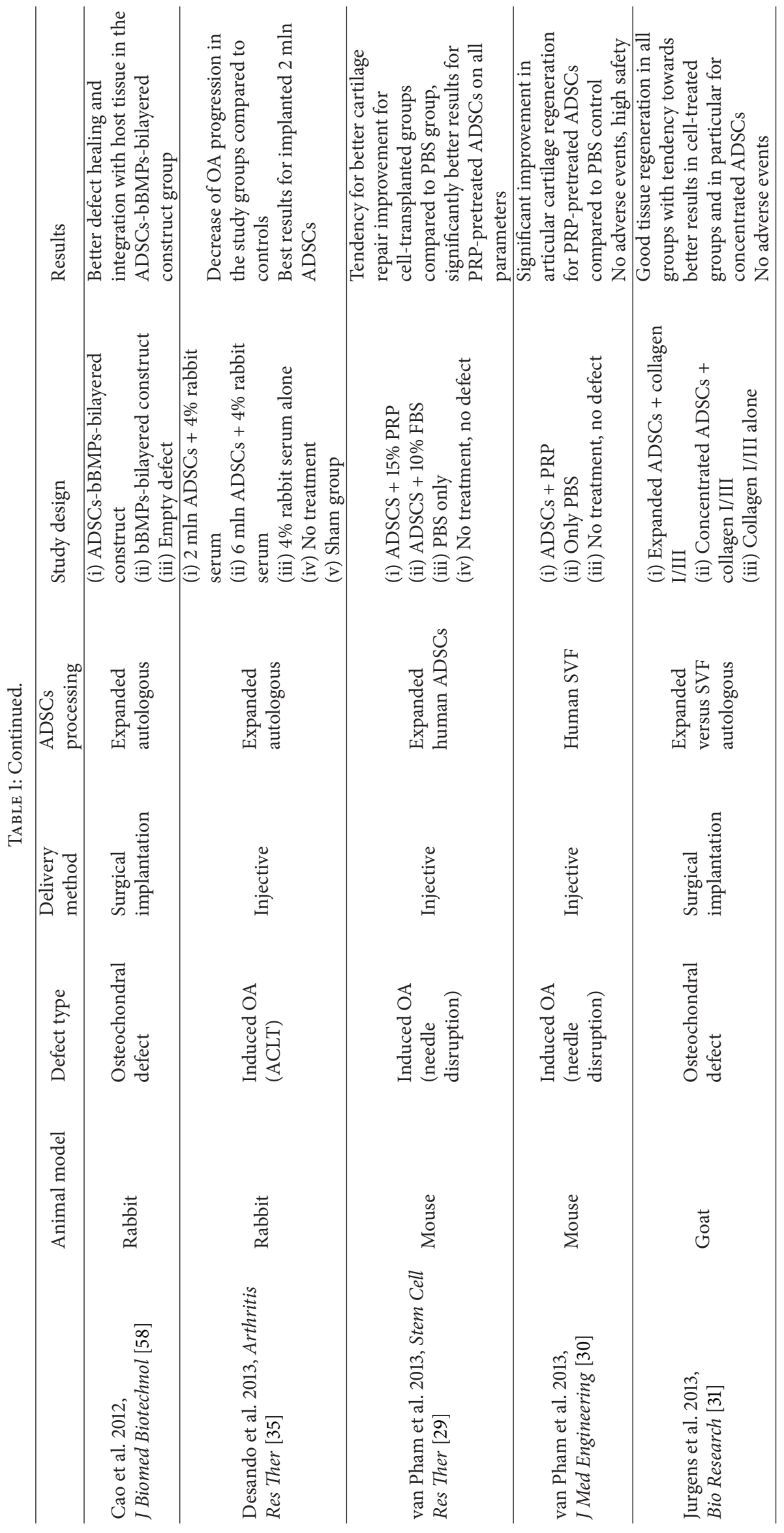




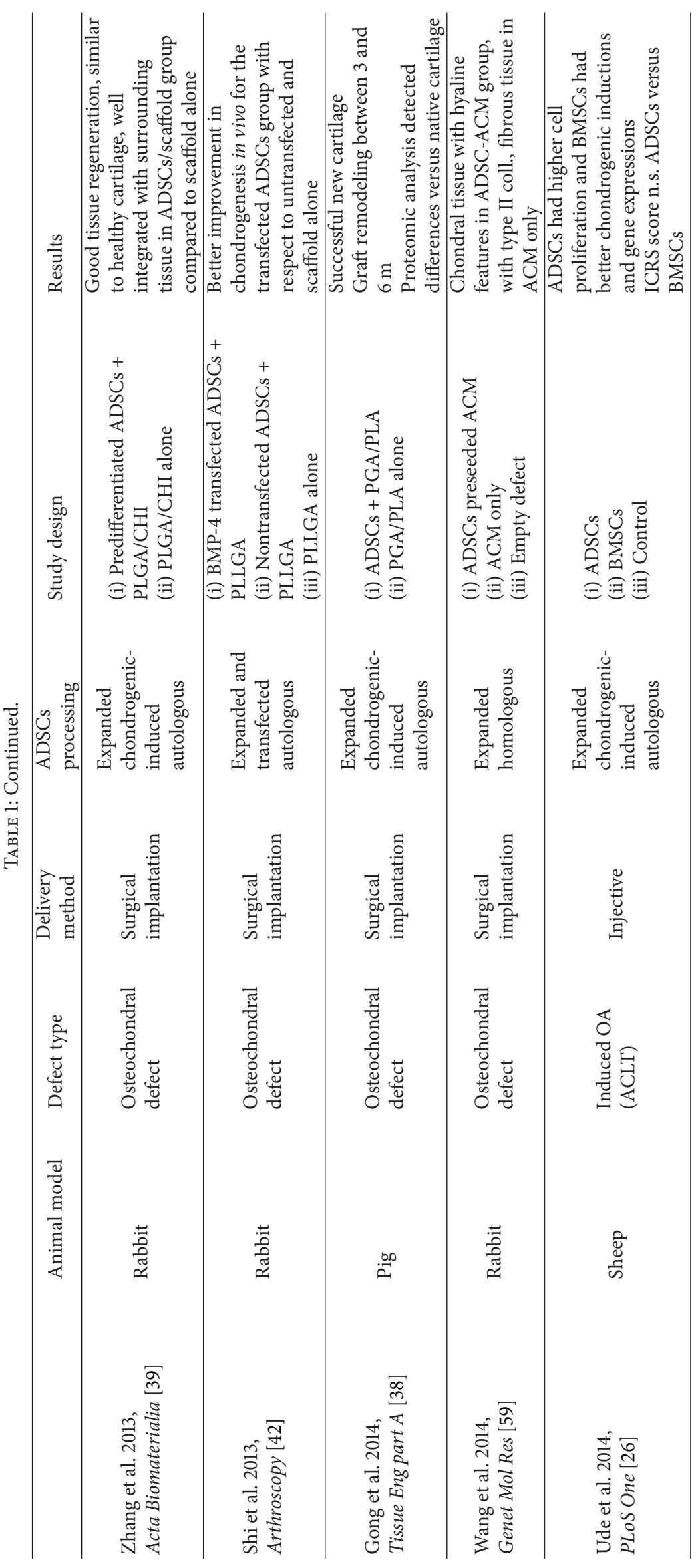




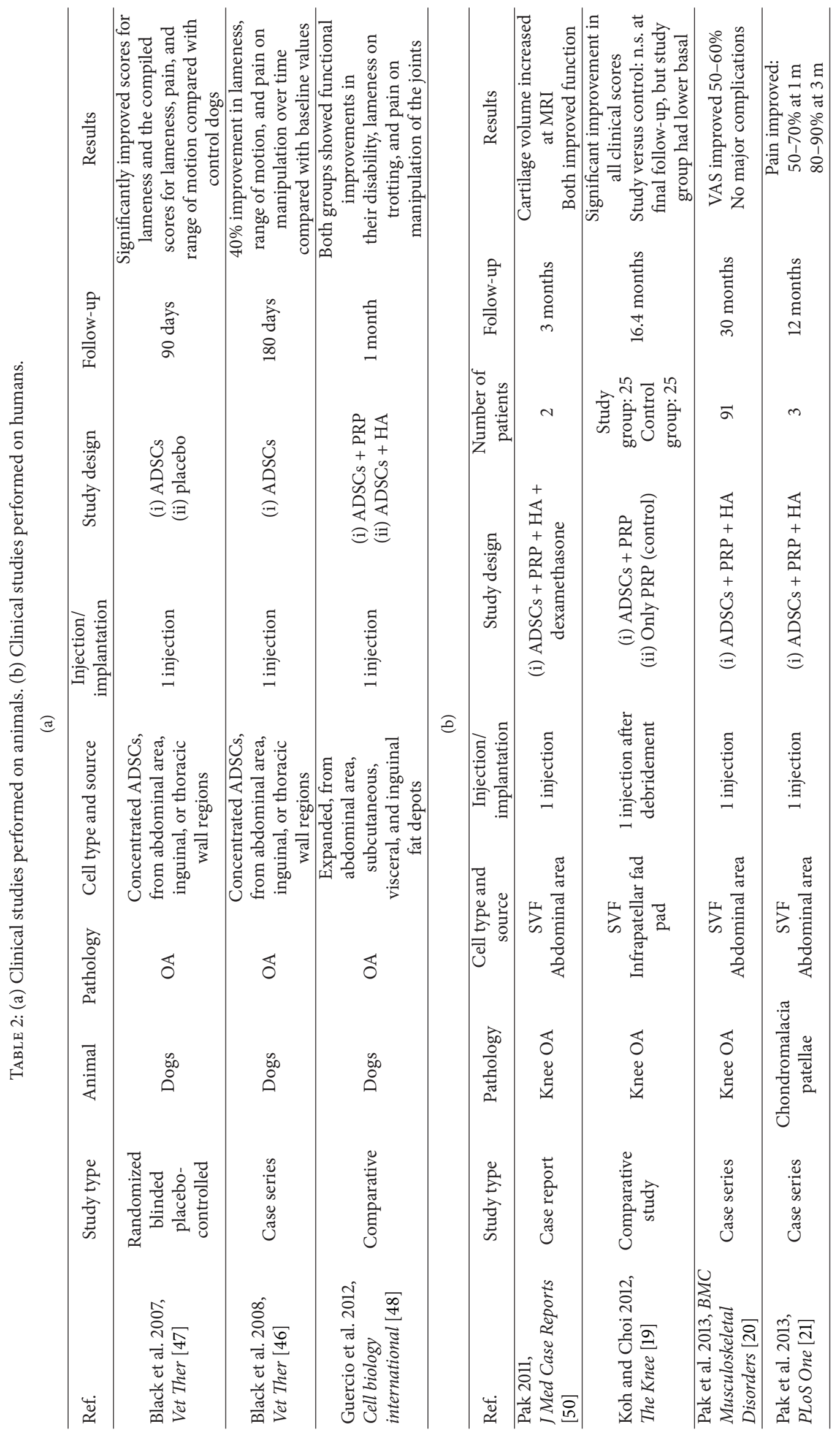




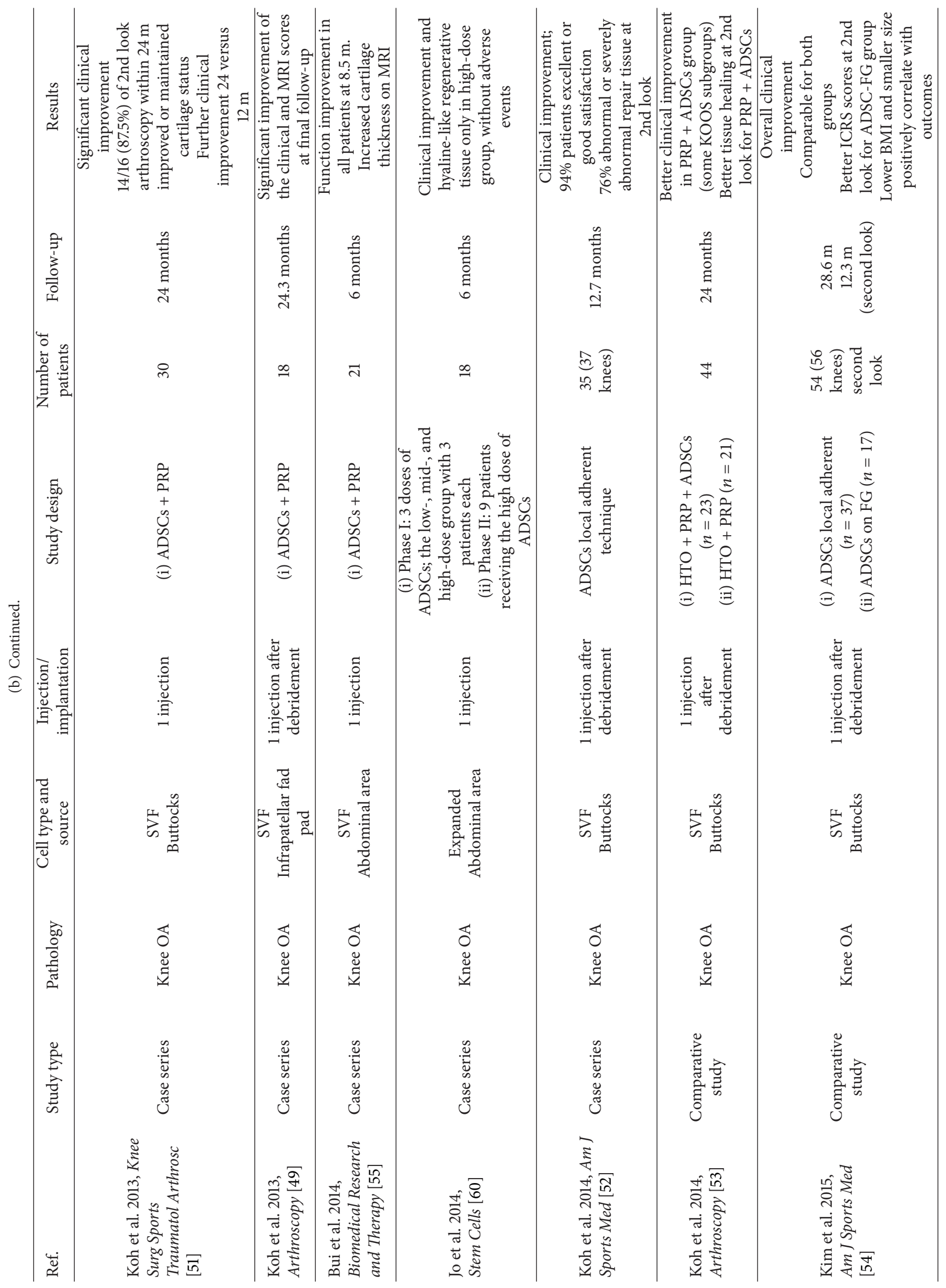


5.1. Mechanism of Action. The regenerative effect of ADSCs was suggested by Nathan and colleagues, who used ADSCs seeded onto a fibrin scaffold for the treatment of osteochondral defects in a rabbit model [22]. Better reconstruction was observed using allogeneic ADSCs compared to using cells from periosteum or a control group [22]. Conversely, three different studies showed poorer outcomes for ADSCs compared to GFs or other cell types in the rabbit model. Im and Lee reported better macroscopic scores by the sole application of GFs (TGF-b2 and BMP-7), with an uncertain effect of adding ADSCs [23], when treating osteochondral defects in rabbits. With regard to cell source, Koga et al. also showed lower cartilage matrix production and lower chondrogenic potential for ADSCs compared with BMSCs and synovial cells [24]. Similar findings were confirmed by $\mathrm{Li}$ et al., who showed the best results for BMSCs in terms of matrix production [25], and more recently by Ude et al., who reported better chondrogenic induction and gene expressions for BMSCs compared to ADSCs [26]. However, no differences were observed in terms of quality of cartilage regeneration in an induced-OA animal model [26]. Frisbie and colleagues reported that injecting cultured cells produces a comparable clinical, radiological, histological, and biochemical improvement, using either BMSC or ADSCs, for the treatment of induced OA in a horse model. However, the authors did not observe significant treatment effects to recommend stem cell treatment for this specific model of OA [27].

All studies except one [27] reported that the administration of ADSCs had a regenerative effect on articular cartilage in the animal model. However, the mechanism of action still remains unclear. Considering which cell may be the best progenitor for hyaline cartilage is still a subject of study [18]; currently, the leading hypothesis is that trophic activity plays a more important role than the intrinsic differentiation potential in the mechanism of action of ADSCs [18, 28], but future studies are needed to clarify this key aspect of ADSCs.

5.2. To Expand or Not to Expand? All except two preclinical trials tested culture-expanded ADSCs and aimed at combining the greatest number of stem cells with various biomaterials for the treatment of critical-size osteochondral defects in the animal model. Two studies used ADSCs in the form of SVF, isolated by the enzymatic digestion of adipose tissue $[29,30]$. van Pham et al. injected ADSC-SVF, previously pretreated with PRP, as a therapy for cartilage injury in mice, which showed improved joint regeneration and the lack of adverse events [30]. Jurgens and colleagues then highlighted the safety and feasibility of this approach by using freshly isolated SVF for one-step osteochondral repair in a goat model [31]. A comparison with expanded ADSCs showed no significant differences between the procedures: after slightly better scores for cultured ADSCs at 4 weeks, the SVF group proceeded towards better cartilage regeneration at the final evaluation. The authors argue that nonexpanded cells might have higher differentiation potential than expanded ones, exploited by a sort of promoting effect on the regenerative process of the other cell types contained in SVF [31].

Certainly, the use of cultured cells allows researchers to isolate and better characterize the desired cell type; however, economic and regulatory issues favor minimal manipulation procedures in the clinical practice. At the present time, the available preclinical literature shows no substantial evidence in favor of any of the two methods, and comparative studies with specific focus are required.

5.3. Injective Therapies for OA: How and When? All 9 preclinical studies dealing with animal OA models reported a positive effect for the intra-articular delivery of ADSCs, with better improvement in the quality of the cartilage with respect to control or sham groups, regardless of the animal model. Toghraie et al. showed the efficacy of injecting ADSCs for induced-OA treatment in a rabbit model $[32,33]$. The effect of a single injection produced significant improvement in cartilage and subchondral bone features, thus delaying the progression of $\mathrm{OA}$, regardless of the source of harvest (infrapatellar fat pad [32] or subcutaneous adipose tissue [33]). Similar findings were observed by Ude et al., who observed regenerative features after a single-dose injection [26], either using induced ADSCs or BMSCs, for the treatment of induced OA in a sheep model. The inhibitory effect of ADSCs on the early stages of OA was confirmed by Ter Huurne and colleagues, who injected cells previously marked with green fluorescent protein (GFP) in a mice model. Interestingly, no effect was observed in late stages of OA [34]. Finally, Desando et al. found that injecting a lower dose of cells $\left(2 \times 10^{6}\right)$ has more beneficial effects than a higher dose $\left(6 \times 10^{6}\right)$ on OA progression, especially in earlier phases of the disease (16 versus 24 weeks) [35]. Thus, both dose and timing of ADSC administration should be considered, even though further studies are needed for a better definition.

5.4. Focal Osteochondral Defect Treatment: Surgical Implantation. Sixteen studies reported the use of ADSCs to treat osteochondral defects in different animal models. Adipose tissue was harvested from subcutaneous tissue in different sites. ADSCs were implanted into the lesions in combination with various biomaterials (ranging from fibrin glue [36] to collagen type I/III matrixes [31], PGA/PLA alone [37, 38] or polymers with chitosan [39], Ca-alginate [40], atelocollagen honeycomb-shaped scaffold with a membrane sealing (ACHMS) [41], polycaprolactone (PCL)/F127 [23], demineralized bone matrix (DBM) [25], or poly(L-lactic-co-glycolic acid) [42]).

Generally, improved tissue regeneration has been highlighted by seeding ADSCs into scaffolds, with good defect repair and the lack of adverse reactions, regardless of the type of biomaterial implanted. Unfortunately, the different biomaterials used and the variability of the study designs prevent any comparison among them.

In conclusion, each technique has shown that it can produce improved outcomes when applied to osteochondral lesions in the animal model, but comparative studies are lacking, and there is no evidence concerning the best treatment modality and the optimal biomaterial to be combined with ADSCs for cartilage regeneration.

5.5. Low Chondrogenic Potential of ADSCs and Improvement Strategies. Some authors tried to overcome the abovementioned relatively low chondrogenic potential of ADSCs 
with different approaches. Shi and colleagues showed that BMP-4 plasmid transfected cells improved chondrogenesis in a PLLGA scaffold in a rabbit model, with respect to nontransfected ADSCs [42]. Lee and Im had similar findings by combining fibrin gel scaffold and cells transduced with SOX-trio proteins in a rat model [43].

Other studies showed the effect of platelet-derived GFs. Xie and colleagues implanted a PRP scaffold seeded with ADSCs or BMSCs in a rabbit model [44]: although BMSCs produced better tissue quality, good outcomes were observed using both cell lines, by having an inductive effect of PRP on MSCs proliferation and cartilage production [44]. Similarly, two studies by van Pham and colleagues tested the administration of human ADSCs, either expanded [30] or isolated as SVF [29], in a mouse OA model. No adverse reactions from the xenogeneic transplant were observed. Moreover, ADSCs pretreated with platelet-derived GFs showed a tendency towards better regeneration in both studies, with no differences between expanded ADSCs or SVF [29, 30]. Finally, one study showed the best defect filling and integration by combining GFs with predifferentiated ADSCs, among other groups with nonpredifferentiated cells or chondrocytes [45].

In conclusion, the regenerative effect of ADSCs can be induced in several ways. The combination of GFs can be a direct and easy method to improve the regenerative cell potential. On the other hand, manipulating ADSCs by introducing inductive genes has also shown improved cell features. Thus, further studies are necessary to determine which method is more effective and whether their combination may be beneficial.

\section{Clinical Findings}

Three clinical animal studies were found, all reporting the results of a single injection of autologous ADSCs for OA treatment in dogs. Two of them used concentrated ADSCs, which showed a significant improvement in all the scores [46], and produced better results than a control group randomly assigned to placebo and blindly evaluated [47]. A further study used expanded cells, combined with either PRP or hyaluronic acid (HA), and reported improved and comparable outcomes 1 month after the injection [48].

Regarding applications in humans, 10 out of the 11 clinical studies reported the use of nonexpanded autologous ADSCs, as SVF. Adipose tissue was obtained by liposuction from the abdominal area or buttocks in all cases, except for two studies where infrapatellar fad pad tissue was harvested during knee arthroscopy [19, 49]. However, the same authors concluded that more ADSCs can be obtained from the buttocks than from infrapatellar fad pad, with the same differentiation potential in both sources [49].

All except three of these clinical papers described ADSCs injected in varying volumes $(3-5 \mathrm{cc}$ ) of autologous PRP, activated with $\mathrm{Ca}$-chloride. Three studies used HA as a carrier instead $[20,21,50]$, and one of them also added dexamethasone to the cell-PRP-HA mixture [50]. After a single injection of ADSCs, a variable number (usually 2) of PRP-only intraarticular injections were used in most of the studies. In four of these studies cells were injected following arthroscopic lavage [51] and debridement $[19,49,52-54]$.
All clinical studies showed that the administration of SVF containing ADSCs improves pain and functional scores at a follow-up of between 3 and 36 months. In three studies MRI analysis was performed, which revealed improved features, including increased cartilage thickness [49, 50, 55]. Four studies evaluated the implant through second-look arthroscopy 6 [36], 12, and 24 months [51, 53, 54] after treatment, which showed maintained or improved joint status in OA patients. However, only one of them performed a histological analysis of the repair tissue, which showed hyaline-like features [55]. Interestingly, the latter study evaluated the effect of different doses of ADSCs and found clinical and histologic improvement and no adverse events only with high-dose administration. Koh and Choi performed the only comparative studies, by testing the effect of ADSCs-PRP (followed by 2 PRP-only injections) with respect to control groups treated with injections of PRP alone [19]. A further study by the same group suggested the beneficial effect of adding fibrin glue to the cells [54].

A preliminary study showed the safety of SVF injections and a significant clinical improvement in both groups [19]. However, no significant difference was found in the outcome, even though the study group (SVF) had significantly lower basal scores [19]. More recently, the same group reported a similar prospective comparison in a group of patients with unicompartmental OA, treated with high tibial osteotomy and randomly assigned to PRP alone or PRP plus SVF injections [53]. At 24-month follow-up, the PRP-SVF group had a better clinical outcome in all KOOS parameters. Whereas statistical significance was reached only in isolated KOOS subgroups, better repair tissue was observed by second look arthroscopy in patients treated with PRP-SVF [53]. A further study on 30 patients found a significant clinical improvement between 12 and 24 months after treatment [51], thus suggesting that the beneficial effect of ADSCs within SVF may be long-lasting. A more recent study used SVF for the treatment of isolated chondral lesions of the knee, as proposed by Koga et al. [56]. Interestingly, the overall significant improvement in terms of clinical scores was negatively affected by higher body mass index (BMI) and larger defect size [52]. Moreover, second look arthroscopic evaluations, performed 1 year after surgery, showed abnormal repair tissue in $76 \%$ of the cases; thus, the authors suggested that the use of scaffolds might further improve the quality of the repair tissue [52]. A partial answer to this question came from a very recent study, where patients with isolated cartilage defects in OA knees were treated with the implantation of ADSCs loaded into fibrin glue (FG) as a scaffold [54]. The clinical and macroscopic outcomes at short-term follow-up were compared with those of patients treated with SVF implantation without scaffold. Besides a comparable clinical and macroscopic improvement in both groups, FG significantly improved the tissue quality (ICRS score) by second look arthroscopy [54]. Finally, higher BMI and larger lesions were confirmed to be negative prognostic factors for the treatment of isolated articular surface defects [54]. Despite the new data arising from their studies, the authors concluded that key concerns, such as the best amount of cells and the ideal scaffold to be used, still remain unsolved. 
All the clinical studies cited above are case series, with several limitations, and heterogeneity in the methods is the main confounding factor in this review. First, no data have been reported concerning the effects of pure SVF-ADSCs injections and since most studies report SVF administered in a volume of PRP, it is impossible to distinguish the effects of ADSCs isolated from those of PRP or other substances [19], which have already been shown to be effective in OA patients $[2,57]$. Similarly, combination with surgical treatments, such as lavage and debridement, provides pain relief in the shortterm [52] and requires further examination. Furthermore, no protocol has yet emerged as the most effective in terms of times and modality of administration. Finally, most of these studies lack a control group, whereas randomized, double blind, and placebo-controlled studies would be key to confirming the efficacy of ADSCs in knee joint disease.

\section{Conclusion}

In conclusion, conversely to the tendency found in the preclinical setting, the injective intra-articular delivery of SVF has emerged as the trend in clinical use. In fact, safety, feasibility, and effectiveness of this approach have been demonstrated at preclinical level, and several aspects favor the use of freshly harvested SVF instead of expanded ADSCs. Concentrating ADSCs would obviate the high costs related to cell manipulation and culture, thus exploiting the increased vitality and differentiation attitude of cells. Moreover, a minimally invasive procedure as injective delivery is more readily accepted by patients due to lower rates of morbidity and adverse events. Furthermore, although the ideal requirement for maximizing the regenerative effect of such therapies would suggest the use of processed cells, there is still lack of consensus about the most effective ADSCs dosage. Currently, the lack of well-designed and comparative studies focusing on the different procedures still leaves many questions unanswered about the mechanism of action of ADSCs, the best way to apply them, and their real therapeutic potential.

\section{Conflict of Interests}

The authors declare that there is no conflict of interests regarding the publication of this paper.

\section{Acknowledgment}

The work leading to this review was partially supported by European Union Seventh Framework Programme FP7PEOPLE-2013-ITN under Grant Agreement no. 607051 and by Italian Ministry of Health, Project "Ricerca Finalizzata2011-02352638".

\section{References}

[1] M. Marcacci, S. Zaffagnini, E. Kon et al., "Unicompartmental osteoarthritis: an integrated biomechanical and biological approach as alternative to metal resurfacing," Knee Surgery, Sports Traumatology, Arthroscopy, vol. 21, no. 11, pp. 2509-2517, 2013.
[2] E. Kon, G. Filardo, M. Drobnic et al., "Non-surgical management of early knee osteoarthritis," Knee Surgery, Sports Traumatology, Arthroscopy, vol. 20, no. 3, pp. 436-449, 2012.

[3] E. Kon, G. Filardo, V. Condello et al., "Second-generation autologous chondrocyte implantation: results in patients older than 40 years," American Journal of Sports Medicine, vol. 39, no. 8, pp. 1668-1675, 2011.

[4] G. Filardo, L. Andriolo, F. Balboni, M. Marcacci, and E. Kon, "Cartilage failures. Systematic literature review, critical survey analysis, and definition," Knee Surgery, Sports Traumatology, Arthroscopy, 2014.

[5] G. Filardo, E. Kon, F. Perdisa et al., "Osteochondral scaffold reconstruction for complex knee lesions: a comparative evaluation," Knee, vol. 20, no. 6, pp. 570-576, 2013.

[6] G. Filardo, F. Vannini, M. Marcacci et al., "Matrix-assisted autologous chondrocyte transplantation for cartilage regeneration in osteoarthritic knees: results and failures at midterm follow-up," American Journal of Sports Medicine, vol. 41, no. 1, pp. 95-100, 2013.

[7] G. Filardo, E. Kon, A. D. Martino et al., "Second-generation arthroscopic autologous chondrocyte implantation for the treatment of degenerative cartilage lesions," Knee Surgery, Sports Traumatology, Arthroscopy, vol. 20, no. 9, pp. 1704-1713, 2012.

[8] E. Kon, G. Filardo, F. Perdisa et al., "A one-step treatment for chondral and osteochondral knee defects: clinical results of a biomimetic scaffold implantation at 2 years of follow-up," Journal of Materials Science: Materials in Medicine, vol. 25, no. 10, pp. 2437-2444, 2014.

[9] G. Filardo, E. Kon, L. Andriolo, B. di Matteo, F. Balboni, and M. Marcacci, "Clinical profiling in cartilage regeneration: prognostic factors for midterm results of matrix-assisted autologous chondrocyte transplantation," The American Journal of Sports Medicine, vol. 42, no. 4, pp. 898-905, 2014.

[10] M. Marcacci, G. Filardo, and E. Kon, “Treatment of cartilage lesions: what works and why?” Injury, vol. 44, supplement 1, pp. S11-S15, 2013.

[11] T. Vos, A. D. Flaxman, M. Naghavi et al., "Years lived with disability (YLDs) for 1160 sequelae of 289 diseases and injuries 1990-2010: a systematic analysis for the Global Burden of Disease Study," The Lancet, vol. 380, no. 9859, pp. 2163-2196, 2012.

[12] K. Yoshimura, T. Shigeura, D. Matsumoto et al., "Characterization of freshly isolated and cultured cells derived from the fatty and fluid portions of liposuction aspirates," Journal of Cellular Physiology, vol. 208, no. 1, pp. 64-76, 2006.

[13] A. M. DiMarino, A. I. Caplan, and T. L. Bonfield, "Mesenchymal stem cells in tissue repair," Frontiers in Immunology, vol. 4, article 201, 2013

[14] L. Wu, X. Cai, S. Zhang, M. Karperien, and Y. Lin, "Regeneration of articular cartilage by adipose tissue derived mesenchymal stem cells: perspectives from stem cell biology and molecular medicine," Journal of Cellular Physiology, vol. 228, no. 5, pp. 938-944, 2013.

[15] G. Filardo, H. Madry, M. Jelic, A. Roffi, M. Cucchiarini, and E. Kon, "Mesenchymal stem cells for the treatment of cartilage lesions: from preclinical findings to clinical application in orthopaedics," Knee Surgery, Sports Traumatology, Arthroscopy, vol. 21, no. 8, pp. 1717-1729, 2013.

[16] P. A. Zuk, M. Zhu, P. Ashjian et al., "Human adipose tissue is a source of multipotent stem cells," Molecular Biology of the Cell, vol. 13, no. 12, pp. 4279-4295, 2002. 
[17] M. Strioga, S. Viswanathan, A. Darinskas, O. Slaby, and J. Michalek, "Same or not the same? comparison of adipose tissuederived versus bone marrow-derived mesenchymal stem and stromal cells," Stem Cells and Development, vol. 21, no. 14, pp. 2724-2752, 2012.

[18] R. A. Somoza, J. F. Welter, D. Correa, and A. I. Caplan, "Chondrogenic differentiation of mesenchymal stem cells: challenges and unfulfilled expectations," Tissue Engineering, Part B: Reviews, vol. 20, no. 6, pp. 596-608, 2014.

[19] Y.-G. Koh and Y.-J. Choi, "Infrapatellar fat pad-derived mesenchymal stem cell therapy for knee osteoarthritis," Knee, vol. 19, no. 6, pp. 902-907, 2012.

[20] J. Pak, J.-J. Chang, J. H. Lee, and S. H. Lee, "Safety reporting on implantation of autologous adipose tissue-derived stem cells with platelet-rich plasma into human articular joints," $B M C$ Musculoskeletal Disorders, vol. 14, article 337, 2013.

[21] J. Pak, J. H. Lee, and S. H. Lee, "A novel biological approach to treat chondromalacia patellae," PLOS ONE, vol. 8, no. 5, Article ID e64569, 2013.

[22] S. Nathan, S. D. De, A. Thambyah, C. Fen, J. Goh, and E. H. Lee, "Cell-based therapy in the repair of osteochondral defects: a novel use for adipose tissue," Tissue Engineering, vol. 9, no. 4, pp. 733-744, 2003.

[23] G.-I. Im and J. H. Lee, "Repair of osteochondral defects with adipose stem cells and a dual growth factor-releasing scaffold in rabbits," Journal of Biomedical Materials Research Part B: Applied Biomaterials, vol. 92, no. 2, pp. 552-560, 2010.

[24] H. Koga, T. Muneta, T. Nagase et al., "Comparison of mesenchymal tissues-derived stem cells for in vivo chondrogenesis: suitable conditions for cell therapy of cartilage defects in rabbit," Cell and Tissue Research, vol. 333, no. 2, pp. 207-215, 2008.

[25] Q. Li, J. Tang, R. Wang et al., "Comparing the chondrogenic potential in vivo of autogeneic mesenchymal stem cells derived from different tissues," Artificial Cells, Blood Substitutes and Biotechnology, vol. 39, no. 1, pp. 31-38, 2011.

[26] C. C. Ude, S. B. Sulaiman, N. Min-Hwei et al., "Cartilage regeneration by chondrogenic induced adult stem cells in osteoarthritic sheep model," PLoS ONE, vol. 9, no. 6, Article ID e98770, 2014.

[27] D. D. Frisbie, J. D. Kisiday, C. E. Kawcak, N. M. Werpy, and C. W. McIlwraith, "Evaluation of adipose-derived stromal vascular fraction or bone marrow-derived mesenchymal stem cells for treatment of osteoarthritis," Journal of Orthopaedic Research, vol. 27, no. 12, pp. 1675-1680, 2009.

[28] M. Ruetze and W. Richter, "Adipose-derived stromal cells for osteoarticular repair: trophic function versus stem cell activity," Expert Reviews in Molecular Medicine, vol. 16, article e9, 2014.

[29] P. van Pham, K. H.-T. Bui, D. Q. Ngo, L. T. Khuat, and N. K. Phan, "Transplantation of nonexpanded adipose stromal vascular fraction and platelet-rich plasma for articular cartilage injury treatment in mice model," Journal of Medical Engineering, vol. 2013, Article ID 832396, 7 pages, 2013.

[30] P. van Pham, K. H.-T. Bui, D. Q. Ngo et al., "Activated plateletrich plasma improves adipose-derived stem cell transplantation efficiency in injured articular cartilage," Stem Cell Research and Therapy, vol. 4, no. 4, article 91, 2013.

[31] W. J. F. M. Jurgens, R. J. Kroeze, B. Zandieh-Doulabi et al., “Onestep surgical procedure for the treatment of osteochondral defects with adipose-derived stem cells in a caprine knee defect: a pilot study," BioResearch Open Access, vol. 2, no. 4, pp. 315-325, 2013.
[32] F. S. Toghraie, N. Chenari, M. A. Gholipour et al., "Treatment of osteoarthritis with infrapatellar fat pad derived mesenchymal stem cells in Rabbit," Knee, vol. 18, no. 2, pp. 71-75, 2011.

[33] F. S. Toghraie, M. Razmkhah, M. A. Gholipour et al., "Scaffoldfree adipose-derived stem cells (ASCs) improve experimentally induced osteoarthritis in rabbits," Archives of Iranian Medicine, vol. 15, no. 8, pp. 495-499, 2012.

[34] M. Ter Huurne, R. Schelbergen, R. Blattes et al., "Antiinflammatory and chondroprotective effects of intraarticular injection of adipose-derived stem cells in experimental osteoarthritis," Arthritis and Rheumatism, vol. 64, no. 11, pp. 3604-3613, 2012.

[35] G. Desando, C. Cavallo, F. Sartoni et al., "Intra-articular delivery of adipose derived stromal cells attenuates osteoarthritis progression in an experimental rabbit model," Arthritis Research and Therapy, vol. 15, no. 1, article R22, 2013.

[36] J. L. Dragoo, G. Carlson, F. McCormick et al., "Healing fullthickness cartilage defects using adipose-derived stem cells," Tissue Engineering, vol. 13, no. 7, pp. 1615-1621, 2007.

[37] L. Cui, Y. Wu, L. Cen et al., "Repair of articular cartilage defect in non-weight bearing areas using adipose derived stem cells loaded polyglycolic acid mesh," Biomaterials, vol. 30, no. 14, pp. 2683-2693, 2009.

[38] L. Gong, X. Zhou, Y. Wu et al., "Proteomic analysis profile of engineered articular cartilage with chondrogenic differentiated adipose tissue-derived stem cells loaded polyglycolic acid mesh for weight-bearing area defect repair," Tissue Engineering Part A, vol. 20, no. 3-4, pp. 575-587, 2014.

[39] K. Zhang, Y. Zhang, S. Yan et al., "Repair of an articular cartilage defect using adipose-derived stem cells loaded on a polyelectrolyte complex scaffold based on poly(l-glutamic acid) and chitosan," Acta Biomaterialia, vol. 9, no. 7, pp. 7276-7288, 2013.

[40] H.-N. Zhang, L. Li, P. Leng, Y.-Z. Wang, and C.-Y. Lü, "Uninduced adipose-derived stem cells repair the defect of fullthickness hyaline cartilage," Chinese Journal of Traumatology, vol. 12, no. 2, pp. 92-97, 2009.

[41] K. Masuoka, T. Asazuma, H. Hattori et al., “Tissue engineering of articular cartilage with autologous cultured adipose tissuederived stromal cells using atelocollagen honeycomb-shaped scaffold with a membrane sealing in rabbits," Journal of Biomedical Materials Research, Part B Applied Biomaterials, vol. 79, no. 1, pp. 25-34, 2006.

[42] J. Shi, X. Zhang, J. Zhu et al., "Nanoparticle delivery of the bone morphogenetic protein 4 gene to adipose-derived stem cells promotes articular cartilage repair in vitro and in vivo," Arthroscopy, vol. 29, no. 12, pp. 2001.e2-2011.e2, 2013.

[43] J. M. Lee and G. I. Im, "SOX trio-co-transduced adipose stem cells in fibrin gel to enhance cartilage repair and delay the progression of osteoarthritis in the rat," Biomaterials, vol. 33, no. 7, pp. 2016-2024, 2012.

[44] X. Xie, Y. Wang, C. Zhao et al., "Comparative evaluation of MSCs from bone marrow and adipose tissue seeded in PRPderived scaffold for cartilage regeneration," Biomaterials, vol. 33, no. 29, pp. 7008-7018, 2012.

[45] J. T. Oliveira, L. S. Gardel, T. Rada, L. Martins, M. E. Gomes, and R. L. Reis, "Injectable gellan gum hydrogels with autologous cells for the treatment of rabbit articular cartilage defects," Journal of Orthopaedic Research, vol. 28, no. 9, pp. 1193-1199, 2010.

[46] L. L. Black, J. Gaynor, C. Adams et al., "Effect of intraarticular injection of autologous adipose-derived mesenchymal stem and 
regenerative cells on clinical signs of chronic osteoarthritis of the elbow joint in dogs," Veterinary Therapeutics, vol. 9, no. 3, pp. 192-200, 2008.

[47] L. L. Black, J. Gaynor, D. Gahring et al., "Effect of adiposederived mesenchymal stem and regenerative cells on lameness in dogs with chronic osteoarthritis of the coxofemoral joints: a randomized, double-blinded, multicenter, controlled trial," Veterinary Therapeutics, vol. 8, no. 4, pp. 272-284, 2007.

[48] A. Guercio, P. Di Marco, S. Casella et al., "Production of canine mesenchymal stem cells from adipose tissue and their application in dogs with chronic osteoarthritis of the humeroradial joints," Cell Biology International, vol. 36, no. 2, pp. 189-194, 2012.

[49] Y.-G. Koh, S.-B. Jo, O.-R. Kwon et al., "Mesenchymal stem cell injections improve symptoms of knee osteoarthritis," Arthroscopy, vol. 29, no. 4, pp. 748-755, 2013.

[50] J. Pak, "Regeneration of human bones in hip osteonecrosis and human cartilage in knee osteoarthritis with autologous adiposetissue-derived stem cells: a case series," Journal of Medical Case Reports, vol. 5, article 296, 2011.

[51] Y.-G. Koh, Y.-J. Choi, S.-K. Kwon, Y.-S. Kim, and J.-E. Yeo, "Clinical results and second-look arthroscopic findings after treatment with adipose-derived stem cells for knee osteoarthritis," Knee Surgery, Sports Traumatology, Arthroscopy, 2013.

[52] Y. G. Koh, Y. J. Choi, O. R. Kwon, and Y. S. Kim, "Second-look arthroscopic evaluation of cartilage lesions after mesenchymal stem cell implantation in osteoarthritic knees," The American Journal of Sports Medicine, vol. 42, no. 7, pp. 1628-1637, 2014.

[53] Y.-G. Koh, O.-R. Kwon, Y.-S. Kim, and Y.-J. Choi, "Comparative outcomes of open-wedge high tibial osteotomy with plateletrich plasma alone or in combination with mesenchymal stem cell treatment: a prospective study," Arthroscopy, vol. 30, no. 11, pp. 1453-1460, 2014.

[54] Y. S. Kim, Y. J. Choi, D. S. Suh et al., "Mesenchymal stem cell implantation in osteoarthritic knees: is fibrin glue effective as a scaffold?" The American Journal of Sports Medicine, vol. 43, no. 1, pp. 176-185, 2015.

[55] K. H. T. Bui, T. D. Duong, N. T. Nguyen et al., "Symptomatic knee osteoarthritis treatment using autologous adipose derived stem cells and platelet-rich plasma: a clinical study," Biomedical Research and Therapy, vol. 1, no. 1, pp. 2-8, 2014.

[56] H. Koga, M. Shimaya, T. Muneta et al., "Local adherent technique for transplanting Mesenchymal Stem Cells as a potential treatment of cartilage defect," Arthritis Research and Therapy, vol. 10, no. 4, article R84, 2008.

[57] G. Filardo, E. Kon, A. Di Martino et al., "Platelet-rich plasma vs hyaluronic acid to treat knee degenerative pathology: study design and preliminary results of a randomized controlled trial," BMC Musculoskeletal Disorders, vol. 13, article 229, 2012.

[58] Z. Cao, S. Hou, D. Sun, X. Wang, and J. Tang, "Osteochondral regeneration by a bilayered construct in a cell-free or cell-based approach," Biotechnology Letters, vol. 34, no. 6, pp. 1151-1157, 2012.

[59] Z. J. Wang, R. Z. An, J. Y. Zhao et al., "Repair of articular cartilage defects by tissue-engineered cartilage constructed with adipose-derived stem cells and acellular cartilaginous matrix in rabbits," Genetics and Molecular Research, vol. 13, no. 2, pp. 4599-4606, 2014.

[60] C. H. Jo, Y. G. Lee, W. H. Shin et al., "Intra-articular injection of mesenchymal stem cells for the treatment of osteoarthritis of the knee: a proof-of-concept clinical trial," Stem Cells, vol. 32, no. 5, pp. 1254-1266, 2014. 

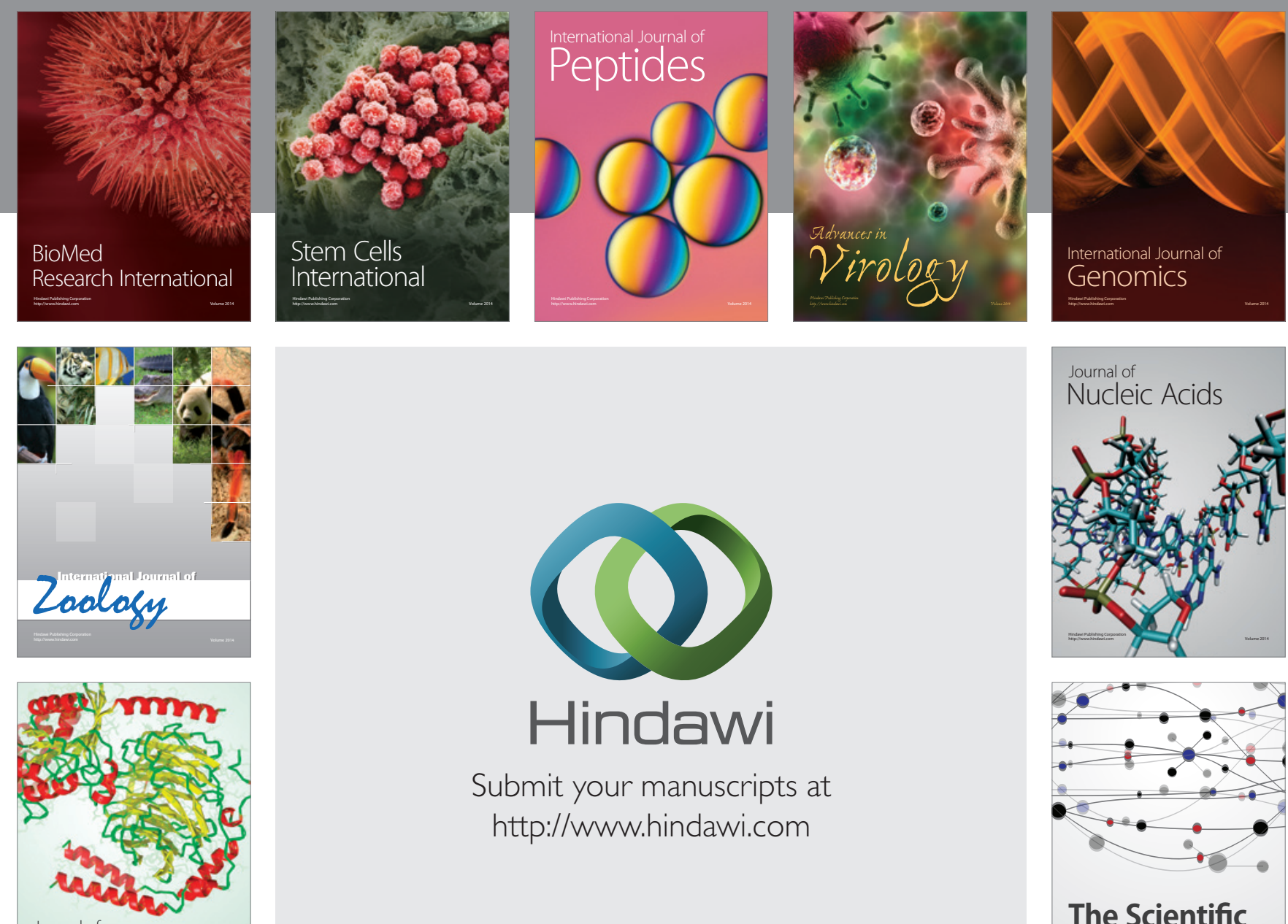

Submit your manuscripts at

http://www.hindawi.com

Journal of
Signal Transduction
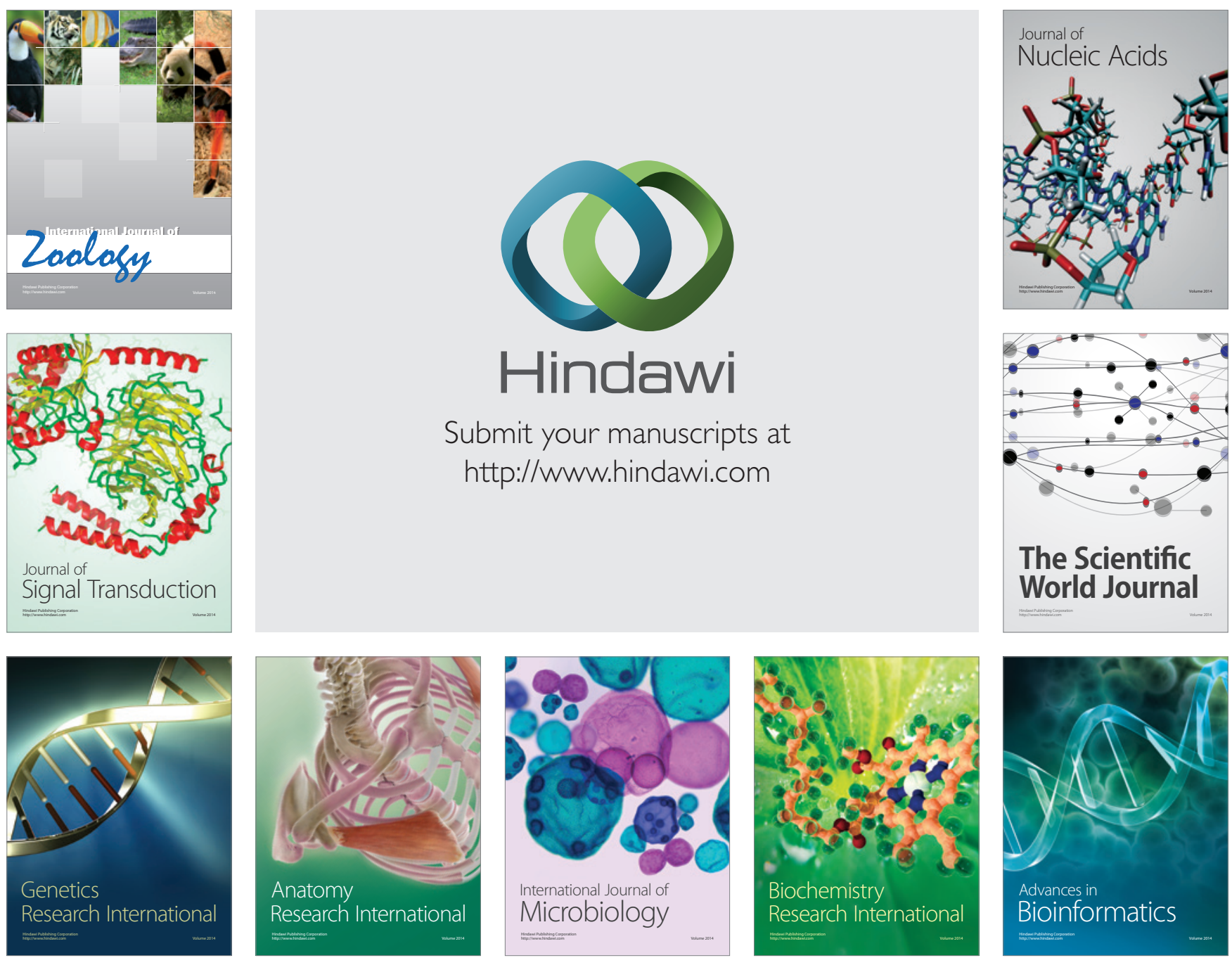

The Scientific World Journal
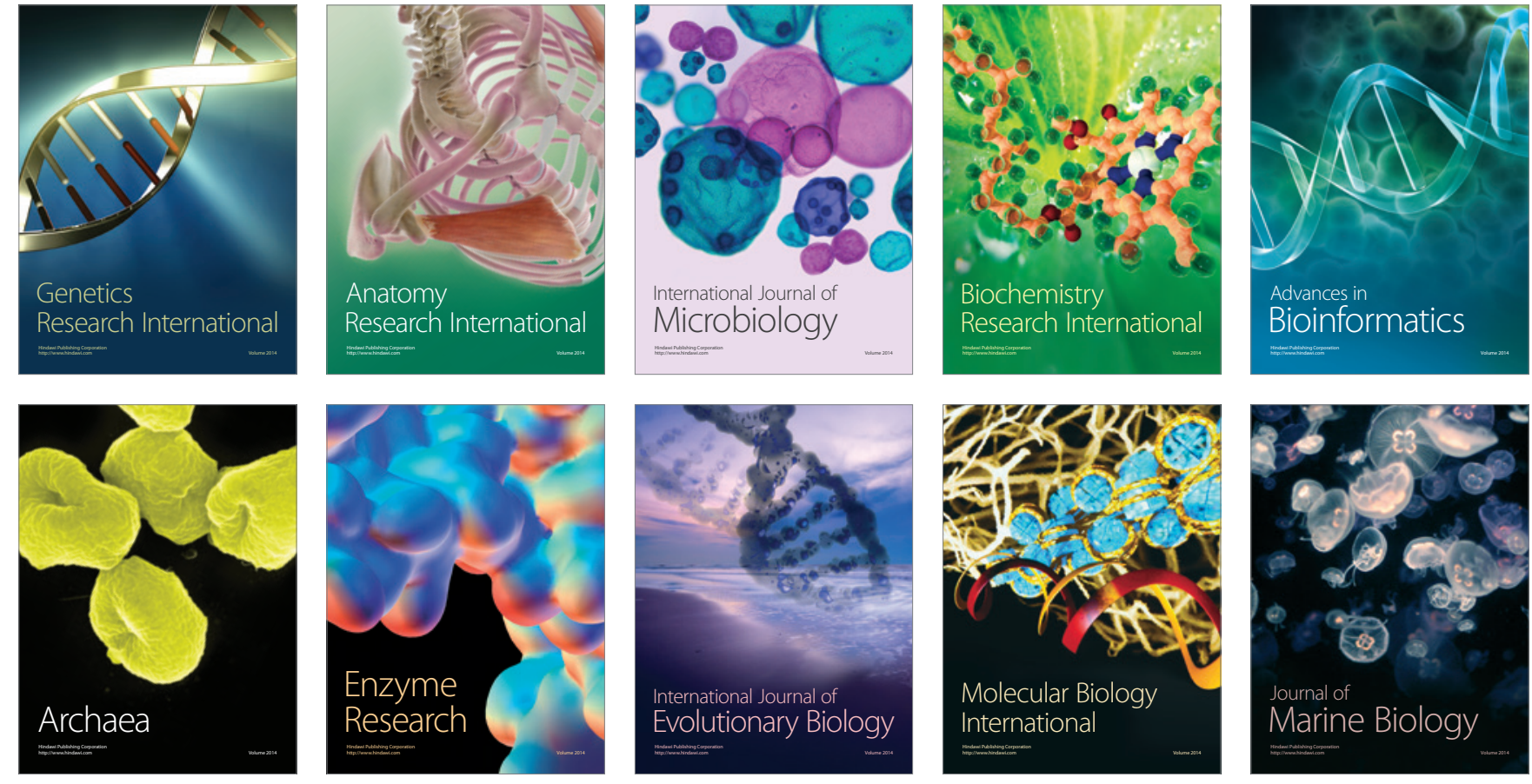